\title{
Learning Co-relations of Plausible Verb Arguments with a WSM and a Distributional Thesaurus*
}

\author{
Hiram Calvo $^{1,2}$, Kentaro Inui ${ }^{2}$, and Yuji Matsumoto ${ }^{2}$ \\ ${ }^{1}$ Center for Computing Research, National Polytechnic Institute, DF, 07738, Mexico \\ ${ }^{2}$ Nara Institute of Science and Technology, Takayama, Ikoma, Nara 630-0192, Japan \\ \{calvo, inui, matsu\} @is.naist.jp
}

\begin{abstract}
We propose a model based on the Word Space Model for calculating the plausibility of candidate arguments given one verb and one argument. The resulting information can be used in co-reference resolution, zero-pronoun resolution or syntactic ambiguity tasks. Previous work such as Selectional Preferences or Semantic Frames acquisition focuses on this task using supervised resources, or predicting arguments independently from each other. On this work we explore the extraction of plausible arguments considering their co-relation, and using no more information than that provided by the dependency parser. This creates a data sparseness problem alleviated by using a distributional thesaurus built from the same data for smoothing. We compare our model with the traditional PLSI method.
\end{abstract}

\section{Introduction}

Several tasks such as co-reference resolution, zero-pronoun resolution or syntactic ambiguity can be regarded as sentence reconstruction tasks that can be solved by measuring the plausibility of each candidate argument. This kind of tasks relies on resources such as semantic frames or selectional preferences for finding the most plausible candidate for a missing part given a context. Consider for example the following sentence:

There is hay at the farm. The cow eats it

We would like to connect it with hay, and not with farm. From selectional preferences we know that the object of eat should be something edible, so that we can say that hay is more edible than farm, solving this issue. From semantic frames, we have similar knowledge, but in a broader sense-there is an ingestor and an ingestible.

However, this information can be insufficient in some cases where the selectional preference depends on other arguments from the clause. For example:

The cow eats hay but the man will eat it

In this case, it is not enough information to know that it should be edible, but also the resolution depends on who is eating. In this case it's unlikely that the man eats hay, so the sentence might refer to the fact that he will eat the cow. The same happens with

\footnotetext{
* We thank the support of Mexican Government (SNI, SIP-IPN, COFAA-IPN, and PIFI-IPN), CONACYT; and the Japanese Government. The second author is currently a JSPS fellow.
} 
other of arguments for verbs. For example, some of the FrameNet peripheral arguments for the ingestion frame are instrument, and place. However, there are some things which are ingested with some instrument - e.g. soup is eaten with a spoon, while rice is eaten with fork, or chopsticks, depending on who is eating; or at different places. Plausible argument extraction allows constructing a database dictionary of this kind of information, which can be regarded as common sense from the fact that it is possible to learn what kind of activities are performed by groups of entities automatically from large blocks of text. See Fig. 1.

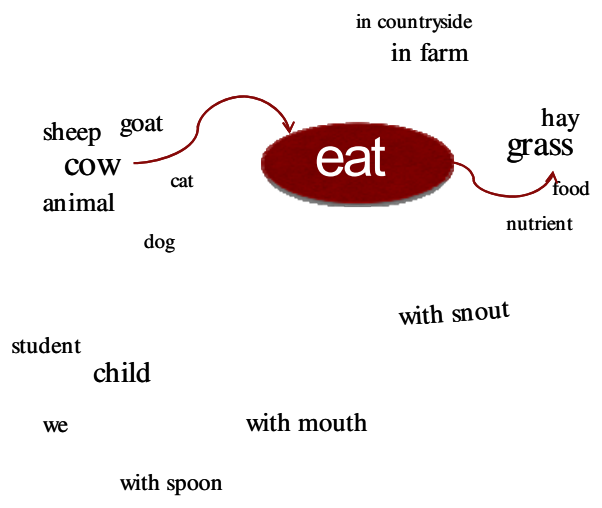

Fig. 1. A verb linking groups of related arguments

The goal of our work is to construct such a database. For this purpose we need to obtain information related to selectional preferences and semantic frames extraction.

Several works are devoted to semantic plausibility extraction, but most of them use supervised resources or consider arguments independently from each other. This work is devoted to the extraction of co-related plausible arguments in an unsupervised way, i.e., no other resource is needed after the dependency parse.

The following section describes work related to verb argument plausibility acquisition, and then we present the results of our experiments within two different approaches. On Section 2.5 we briefly discuss some possible applications, on Section 3 we evaluate our approach and finally we draw or conclusions.

\section{Related Work}

The problem of automatic verb argument plausibility acquisition can be studied from several points of view. From the viewpoint of the kind of information extracted we can find related work for selectional preferences and semantic frames extraction. From the approach of selectional preferences, the task is focused on automatically obtaining classes of arguments for a given verb and a syntactic construction. From the approach of semantic frames, arguments are grouped by the semantic role they have, regardless of the syntactic construction they have. This latter approach emphasizes the distinction between core (indispensable) or peripheral arguments. On the other hand, 
we can consider the viewpoint of how this information is represented: the task can be regarded as a case of statistic language modeling, where given a context — verb and other arguments, the missing argument should be inferred with high probability; or it can be regarded as a word space model task frequently seen in IR systems. In the next sections we present works related to this task from those different viewpoints.

\subsection{Selectional Preferences}

Selectional preferences acquisition can be regarded as one of the first attempts to automatically find argument plausibility. Early attempts dealt with simpler <verb, argument $>$ pairs. Since the learning resource is sparse, all of these works use a generalization, or smoothing mechanism for extending coverage. [16] uses WordNet for generalizing the object-argument. [1] use a class-to-class model, so that the both verb as well as the object-argument are generalized by belonging to a class using WordNet. [11] acquire selectional preferences as probability distributions over the WordNet noun hyponym hierarchy. They use other argument relationships aside from objectargument. [13] combine semantic and syntactic information by estimating his model using corpora with semantic role annotation (i.e. FrameNet, PropBank), and then applying class-based smoothing using WordNet. They model the plausibility of a verb and argument in a given role as

Plausibility $_{v, r, a}=P(v, s, g f, r, a)=P(v) \cdot P(s \mid v) \cdot P(g f \mid v, s) \cdot P(r \mid v, s, g f) \cdot P(a \mid v, s, g f, r)$,

where $P(s \mid v)$ is the probability of a particular sense of a verb, $P(g f v, s)$ is the syntactic subcategorizations of a particular verb sense, $P(r \mid v, s, g f)$ reflects how the verb prefers to realize its thematic role fillers syntactically and $P(a \mid v, s, g f, r)$ is verb's preference for certain argument types and estimate the fit of a verb and argument in a given role.

\subsection{Subcategorization Frames}

The following works deal with the problem of semisupervised argument plausibility extraction from the subcategorization frames extraction approach. [17] acquire verb argument structures. They generalize nouns by using a Named Entity Recognizer (IdentiFinder) and then they use the noisy channel framework for argument prediction. Example of the kind of information they are working with are: Organization bought organization from organization, Thing bought the outstanding shares on date, and, sometimes without generalization, The cafeteria bought extra plates.

Another semi-supervised work is [9]. They generalize by using a manually created thesaurus. For finding case frames they use together with the verb, the closest argument, providing verb sense disambiguation for cases similar as the example which motivated us, presented in Section 1.

Next we discuss two different viewpoints for dealing with the verb argument information representation.

\subsection{Language Modeling}

We can regard the task of finding the plausibility of a certain argument for a set of sentences as estimating a word given an specific context. Particularly for this work we can consider context as the grammar relationships for a particular verb: 


$$
P(w, c)=P(c) \cdot P(c \mid w)
$$

which can be estimated in many ways, particularly, using a hidden markov model, or using latent variables for smoothing, for PLSI [8]:,

$$
P(w, c)=\sum_{Z_{i}} P(z) \cdot P(w \mid z) \cdot P(c \mid z)
$$

The conditional probability can be calculated from n-gram frequency counts.

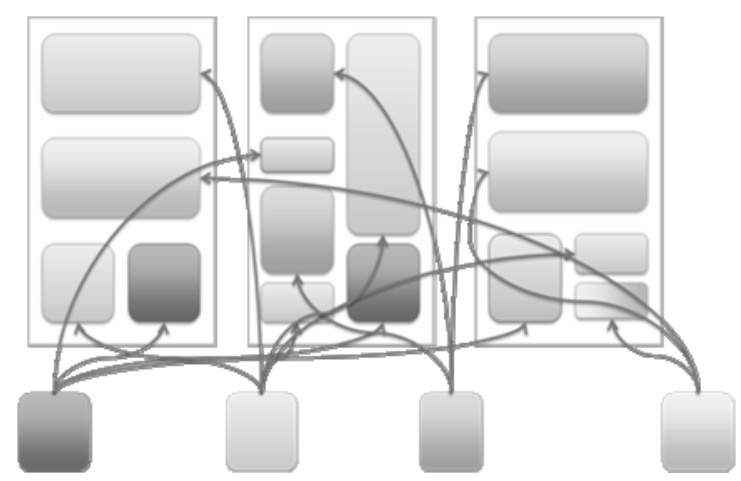

Fig. 2. Each document in PLSI is represented as a mixture of topics

\subsection{Word Space Model}

Traditionally from Information Retrieval, words can be represented as documents and semantic context as features, so that it is possible to build a co-occurrence matrix, or word space, where each intersection of word and context shows the frequency count of each number. This approach has been recently used with syntactic relationships [13]. An important issue within this approach is the similarity measure chosen for comparing words (documents) given its features. Popular similarity measures range from simple measures such as Euclidean distance, cosine and Jaccard's coefficient [10], to measures such as Hindle's measure and Lin's measure.

\subsection{Potential Applications}

Since Resnik [16], selectional preferences have been used in a wide range of applications: Improving parsing, since it is possible to disambiguate syntactic structures if we know the kind of arguments expected for a sentence [4]; Inference of meaning of unknown words-Uttering I eat borogoves makes us think that a borogove might be edible; Co-reference resolution [15]; Word Sense Disambiguation [11, 12]; Metaphora recognition, an uncommon usage of an argument would make a sentence odd, thus, perhaps containing a metaphora (or a coherence mistake); Semantic Plausibility [14], Malapropism detection [2, 3] "hysteric center", instead of historic center, "density has brought me to you", instead of destiny. 


\section{Methodology}

We propose a model based on the Word Space Model. Since we want to consider argument co-relation, we use the following information:

$P\left(v, r_{l}, n_{1}, r_{2}, n_{2}\right)$, where $v$ is a verb, $r_{l}$ is the relationship between verb and $n_{l}$ (noun) as subject, object, preposition or adverb. $r_{2}$ and $n_{2}$ are analogous. If we assume that $n$ has a different function when used with another relationship, then we can consider that $r$ and $n$ form a new symbol, called $a$. So that we can simplify our 5-tuple to $P\left(v, a_{1}, a_{2}\right)$. We want to know, given a verb and an argument $a_{1}$, which $a_{2}$ is the most plausible, we can write this as $P\left(a_{2} \mid v, a_{1}\right)$. For PLSI this can be estimated by

$$
P\left(a_{2}, v, a_{1}\right)=\operatorname{Sum}\left(Z_{i}, P(z) \cdot P\left(a_{2} \mid z\right) \cdot P\left(v, a_{1} \mid z\right)\right. \text {. }
$$

For the word space model, we can build a matrix where $a_{2}$ are the rows (documents) and $v, a_{1}$ are features. As this matrix is very sparse, we use a thesaurus for smoothing the argument values. For doing this, we loosely followed the approach proposed by [12] for finding the predominant sense, but in this case we use the $k$ nearest neighbors of each argument $a_{i}$ to find the prevalence score of an unseen triple given its similarity to all triples present in the corpus, measuring this similarity between arguments. In other words, as in $[12,18,19]$ for WSD, each similar argument votes for the plausibility of each triple.

$$
\begin{aligned}
& \text { Prevalence }\left(v, x_{1}, x_{2}\right)= \\
& \frac{\sum_{<v, a_{1}, a_{2}>\in T} \operatorname{sim}\left(a_{1}, x_{1}\right) \cdot \operatorname{sim}\left(a_{2}, x_{2}\right) \cdot P_{M L E}\left(v, a_{1}, a_{2}\right)}{\sum_{<v, a_{1}, a_{2}>\in T} \operatorname{sim} \_ \text {exists }\left(a_{1}, a_{2}, x_{1}, x_{2}\right)}
\end{aligned}
$$

where $T$ is the whole set of < verb, argument $_{1}$, argument $_{2}>$ triples and

$$
\begin{aligned}
& \text { sim_exists }\left(a_{1}, a_{2}, x_{1}, x_{2}\right)= \\
& \left\{\begin{array}{lc}
1 & \text { if } \operatorname{sim}\left(a_{1}, x_{1}\right) \cdot \operatorname{sim}\left(a_{2}, x_{2}\right)>0 \\
0 & \text { otherwise }
\end{array}\right.
\end{aligned}
$$

For measuring the similarity between arguments, we built a thesaurus using the method described by [5], using the Minipar browser [6] over short-distance relationships, i.e., we previously separated subordinate clauses. We obtained triples $\left\langle v, a_{1}, a_{2}\right\rangle$ from this corpus, which were counted, and these were used for both building the thesaurus as well as a source of verb and argument co-occurrences.

\subsection{Evaluation}

We compared these two models in a pseudo-disambiguation task following [20]. First we obtained triples $\left\langle v, a_{1}, a_{2}\right\rangle$ from the corpus. Then, we divided the corpus in training $(80 \%)$ and testing $(20 \%)$ parts. With the first part we trained the PLSI model and created the WSM. This WSM was also used for obtaining the similarity measure for 
every pair of arguments $a_{2}, a_{2}^{\prime}$. Then we are able to calculate Plausibility $\left(v, a_{1}, a_{2}\right)$. For evaluation we created artificially 4tuples: $\left\langle v, a_{1}, a_{2}, a_{2}^{\prime}\right\rangle$, formed by taking all the triples $\left\langle v, a_{1}, a_{2}\right\rangle$ from the testing corpus, and generating an artificial tuple $\left\langle v, a_{1}, a_{2}^{\prime}\right\rangle$ choosing a random $a_{2}^{\prime}$ with $r_{2}^{\prime}=r_{2}$, and making sure that this new random triple $\left\langle v, a_{1}, a_{2}^{\prime}\right\rangle$ was not present in the training corpus. The task consisted on selecting the correct tuple.

We compared two models based on the Statistical Language Model and the Word Space Model approaches respectively. Using the patent corpus from the NII Test Collection for Information Retrieval System, NTCIR-5 Patent [7], we parsed 7300 million tokens, and then we extracted the chain of relationships on a directed way, that is, for the sentence: $\mathrm{X}$ add $\mathrm{Y}$ to $\mathrm{Z}$ by $\mathrm{W}$, we extracted the triples: $\langle a d d, s u b j-X, o b j-Y>$, $<a d d$, obj-Y, to-Z>, $<a d d$, to-Z, by-W>. We obtained $706 \mathrm{M}$ triples in the form $<v, a_{1}$, $a_{2}>$. We considered only chained asymmetric relationships to avoid false similarities between words co-occurring in the same sentence.

Following [20], we chose 20 verbs, covering high-frequency verbs and lowfrequency verbs and for each one we extracted all the triples $\left\langle v, a_{1}, a_{2}>\right.$ present in the triples corpus. Then we performed experiments with the PLSI algorithm, and the WSM algorithm.

We experimented with different number of topics for the latent variable $z$ in PLSI, and with different number of neighbors from the Lin thesaurus for expanding the WSM. Results are shown in Table 1 for individual words, 10 neighbors for WSM and 10 topics for PLSI. Figure 3 shows average results for different neighbors and topics.

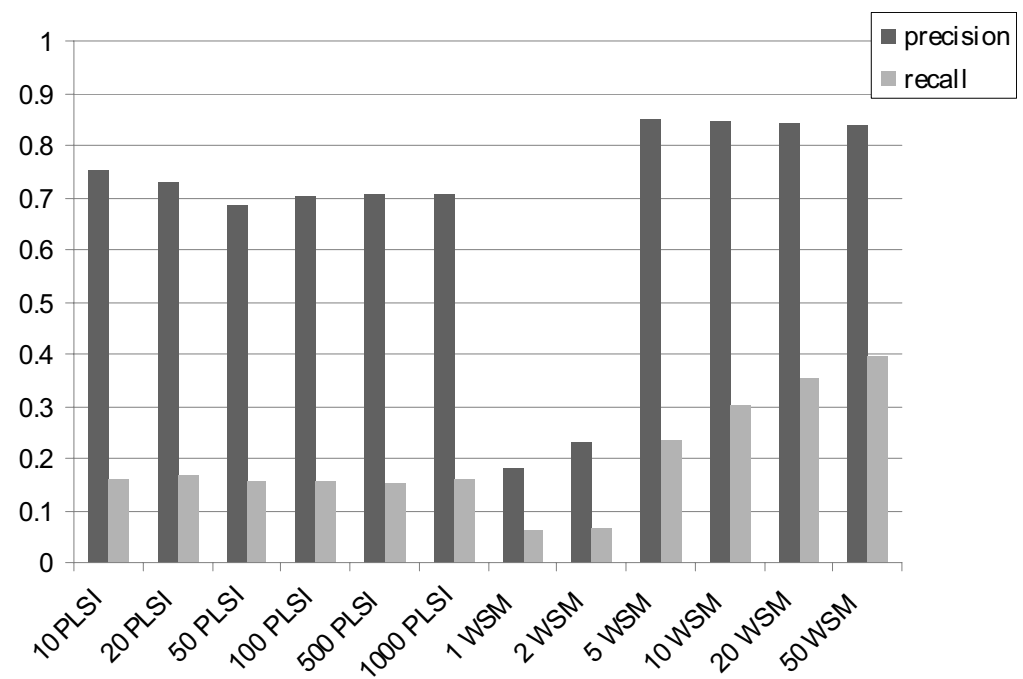

Fig. 3. Results for (topics)-PLSI and (neighbors)-WSM 


\section{Conclusions}

We have proposed a new algorithm within the WSM approach for unsupervised plausible argument extraction and compared with a traditional PLSI approach, obtaining particular evidence to support that it is possible to achieve better results with the method which votes for common triples using a distributional thesaurus. The results look consistent with previous works using distributional thesauri $[4,18,19]$ (see Figure 3 ): adding information increases coverage with little sacrifice on precision. We should experiment with the upper limit of the increasing coverage, as each neighbor from the thesaurus is adding noise. We have experimented with building the thesaurus using the same corpus; however, significant differences could be found if using an encyclopedia corpus for building the dictionary, as broader and richer context could be found.

We call our work unsupervised because we are not using any other resource after the dependency parser, such as named entity recognizers, or labeled data used for training a machine learning algorithm. As a future work we plan to improve the overall recall measure by adding a back-off technique, which could consist simply on considering the information based on the verb when information of the verb and one argument is not available. We plan also to explore specific applications for this algorithm.

Table 1. Precision (P) and Recall (R) for each verb for 10 neighbors (WSM) and 10 topics (PLSI)

\begin{tabular}{lrllll}
\hline verb & \multicolumn{2}{c}{ triples } & \multicolumn{2}{c}{ WSM-10 } & \multicolumn{2}{c}{ PLSI-10 } \\
& & P & R & P & R \\
\hline eat & 31 & 0.98 & 0.92 & 1.00 & 0.04 \\
seem & 77 & 0.88 & 0.09 & 0.64 & 0.38 \\
learn & 204 & 0.82 & 0.10 & 0.57 & 0.22 \\
inspect & 317 & 0.84 & 0.19 & 0.43 & 0.12 \\
like & 477 & 0.79 & 0.13 & 0.54 & 0.24 \\
come & 1,548 & 0.69 & 0.23 & 0.78 & 0.17 \\
play & 1,634 & 0.68 & 0.18 & 0.69 & 0.19 \\
go & 1,901 & 0.81 & 0.25 & 0.80 & 0.15 \\
do & 2,766 & 0.80 & 0.24 & 0.77 & 0.19 \\
calculate & 4,676 & 0.91 & 0.36 & 0.81 & 0.13 \\
fix & 4,772 & 0.90 & 0.41 & 0.80 & 0.13 \\
see & 4,857 & 0.76 & 0.23 & 0.84 & 0.20 \\
write & 6,574 & 0.89 & 0.31 & 0.82 & 0.15 \\
read & 8,962 & 0.91 & 0.36 & 0.82 & 0.11 \\
add & 15,636 & 0.94 & 0.36 & 0.81 & 0.10 \\
have & 127,989 & 0.95 & 0.48 & 0.89 & 0.03 \\
\hline \multicolumn{1}{c}{ average } & 11,401 & 0.85 & 0.30 & 0.75 & 0.16 \\
\hline
\end{tabular}




\section{References}

1. Agirre, E., Martinez, D.: Learning class-to-class selectional preferences. In: Workshop on Computational Natural Language Learning, ACL (2001)

2. Bolshakov, I.A., Galicia-Haro, S.N., Gelbukh, A.F.: Detection and Correction of Malapropisms in Spanish by Means of Internet Search. In: Matoušek, V., Mautner, P., Pavelka, T. (eds.) TSD 2005. LNCS (LNAI), vol. 3658, pp. 115-122. Springer, Heidelberg (2005)

3. Budanitsky, E., Graeme, H.: Semantic distance in WorldNet: An experimental, application-oriented evaluation of five measures. In: NAACL Workshop on WordNet and other lexical resources (2001)

4. Calvo, H., Gelbukh, A., Kilgarriff, A.: Automatic Thesaurus vs. WordNet: A Comparison of Backoff Techniques for Unsupervised PP Attachment. In: Gelbukh, A. (ed.) CICLing 2005. LNCS, vol. 3406, pp. 177-188. Springer, Heidelberg (2005)

5. Lin, D.: Automatic Retrieval and Clustering of Similar Words. In: Procs. 36th Annual Meeting of the ACL and 17th International Conference on Computational Linguistics (1998)

6. Lin, D.: Dependency-based Evaluation of MINIPAR. In: Proc. Workshop on the Evaluation of Parsing Systems (1998)

7. Fujii, A., Iwayama, M. (eds.): Patent Retrieval Task (PATENT). Fifth NTCIR Workshop Meeting on Evaluation of Information Access Technologies: Information Retrieval, Question Answering and Cross-Lingual Information Access (2005)

8. Hoffmann, T.: Probabilistic Latent Semantic Analysis, Uncertainity in Artificial Intelligence, UAI (1999)

9. Kawahara, D., Kurohashi, S.: Japanese Case Frame Construction by Coupling the Verb and its Closest Case Component. In: 1st Intl. Conf. on Human Language Technology Research, ACL (2001)

10. Lee, L.: Measures of Distributional Similarity. In: Procs. 37th ACL (1999)

11. McCarthy, D., Carroll, J.: Disambiguating Nouns, Verbs, and Adjectives Using Automatically Acquired Selectional Preferences. Computational Linguistics 29(4), 639-654 (2006)

12. McCarthy, D., Koeling, R., Weeds, J., Carroll, J.: Finding predominant senses in untagged text. In: Procs. 42nd meeting of the ACL, pp. 280-287 (2004)

13. Padó, S., Lapata, M.: Dependency-Based Construction of Semantic Space Models. Computational Linguistics 33(2), 161-199 (2007)

14. Padó, U.M., Crocker, F., Keller, F.: Modelling Semantic Role Plausibility in Human Sentence Processing. In: Procs. EACL (2006)

15. Ponzetto, P.S., Strube, M.: Exploiting Semantic Role Labeling, WordNet and Wikipedia for Coreference Resolution. In: Procs. Human Language Technology Conference, NACACL, pp. 192-199 (2006)

16. Resnik, P.: Selectional Constraints: An Information-Theoretic Model and its Computational Realization. Cognition 61, 127-159 (1996)

17. Salgueiro, P., Alexandre, T., Marcu, D., Volpe Nunes, M.: Unsupervised Learning of Verb Argument Structures. In: Gelbukh, A. (ed.) CICLing 2006. LNCS, vol. 3878, pp. 59-70. Springer, Heidelberg (2006)

18. Tejada, J., Gelbukh, A., Calvo, H.: An Innovative Two-Stage WSD Unsupervised Method. SEPLN Journal 40 (March 2008)

19. Tejada, J., Gelbukh, A., Calvo, H.: Unsupervised WSD with a Dynamic Thesaurus. In: 11th International Conference on Text, Speech and Dialogue. TSD 2008, Brno, Czech Republic, September 8-12 (2008)

20. Weeds, J., Weir, D.: A General Framework for Distributional Similarity. In: Procs. conf. on EMNLP, vol. 10, pp. 81-88 (2003) 